\title{
Platelet rich plasma
}

\author{
N.Priya
}

\begin{abstract}
Platelet rich plasma is an autologous concentration of eight growth factors PDGF-AA, $P D G F-B B, P D G F-A B$,Transforming growth factor $\beta 1$ and $\beta 2$, vascular endothelial growth factor $(V E G F)$, epidermal growth factor $(E G F)$, and insulin like growth factor.
\end{abstract}

Nowadays PRP is widely used in all the fields of dentistry. This article explains the various potential clinical applications .PRP is prepared from the patient's own blood and there is a less risk of transmission of diseases.

Key Words:PRP, platelets, growth factors, bone regeneration.

\section{INTRODUCTION}

Platelet rich plasma is new and potentially useful adjunct in oral and maxillofacial bone reconstructive surgery, platelet are essential in the wound healing process initiate coagulation by providing primary haemostasis.(1). They release multiple wound healing growth factors and cytokines, including platelet derived growth factors (PDGF), transforming growth factor (TGF), vascular endothelial factor (VEGF), platelet derived endothelial growth factor (PDEGF), interleukin -1, basic fibroblast growth factor (BFGF) and platelet activating growth factor.(2) wirthlin (3)defined growth factors as naturally occurring polypeptide molecules, some what like hormones in structure and function but potent local activity rather than systemic effects.

Platelet derived growth factor is glycoprotein of molecular weight approximately $30 \mathrm{kda}$. It seems to be the first growth factor present in a wound ,and it initiates connective tissue healing including bone regeneration and repair.PDGF accelerates early wound closure via enhanced glygosaminoglycan, hyaluronic acid and fibrinectin deposition.(4) There are about 1200 molecules of PDGF in an individual growth factor (5) Most specific activities of PDGF include mitogenesis, angiogenesis and macrophage activation .PDGF also, stimulates cell replication of important stem cells for fibroblasts and endothelial cells, stimulates production of fibrinectin and hyaluronic acid and helps bring about wound contraction and remodelling (6).

TGF beta inhibit osteoclasts formation and bone resorption thus favouring bone formation over resorption by different mechanisms (5). The most important functions of TGF $\beta 1$ And TGF $\beta$ chemo taxis and mitogenesis of osteoblasts precursors. They have the ability to stimulate osteoblasts deposition of collagen matrix of wound healing and bone. These factors favour bone formation by enlarging the rate of stem cell proliferation.(7)

PDEGF was discovered by Cohen in 1962 and was first growth factor described . It stimulated epidermal regeneration ,promotes wound healing by stimulating the proliferation of keratinocytes and dermal fibroblast, and enhances the effects and production of other growth factor (8). PDAF (6)has the capacity to induce vascularisation. It stimulates vascular endothelial cells by direct or indirect actions and is involved in the process by which blood vessels invaded vascularised tissues .several growth factors and cytokines up regulate PDAF including IGF -1,TGF $\alpha$ and $\beta$,PDGF ,PDEGF,BFGF and IL- $\beta$

\section{PREPARATION OF AUTOLOGOUS PLATELET CONCENTRATE / PRP}

The preparation of autologous platelet concentrate otherwise known platelet rich plasma Can be accomplished using a gradient density cell separator or by the use of a Centrifugation(9).

On the day of surgery $15 \mathrm{ml}$ of blood was drawn from the patient .Blood was Collected in sterile glass tubes containing $3.8 \%$ sodium citrate as an anti coagulant.

The test tube were gently shaken to facilitate anti coagulation $.3 \mathrm{ml}$ was used to obtain a complete blood picture of the patient. The remaining $12 \mathrm{ml}$ was centrifuged following a 2 step procedure. The initial centrifugation process was done at $5000 \mathrm{rpm}$ for 10 minutes. This resulted in separation of 3 basic fractions from least dense to most (10).

1.Platelet poor plasma on the top of the preparation which contains few platelets (11). 2. Middle layer comprising of platelet rich plasma which consists of platelets and white blood cells (buffy coat) 
3. The bottom most fraction comprising of red blood corpuscles which also contains

Newly synthesized platelets at the top.

PRP is plasma with a concentrated number Of platelets and few white blood cells $(12)$

Plasma along with the top erythrocyte layer is collected using a 21 gauge spinal needle .A

spinal needle being long is ideally suited for aspiration of the needle and 21 gauge Helps to avoid sequestration of the platelets. This was then transferred to a empty vacutainer tube after removing the anticoagulant within it and centrifuged For 10 minutes at $2000 \mathrm{rpm}$ The reduced speed is aimed at a more precise separation of the PRP from RBC layer coagulation is achieved by adding bovine thrombin and calcium chloride to produce a gel like consistency. $(13,14)$

\section{Clinical Applications of PRP in dentistry}

\section{Effect of PRP on healing the alveolar socket after tooth extraction.}

Use of PRP in periodontal defect treatment after extraction of impacted third molars. Gilberto sammartino (15) showed that PRP is effective in inducing and accelerating bone regeneration for the treatment of periodontal defects at the distal root of the mandibular second molar after surgical extraction of a mesioangular deeply impacted third molars. The study was conducted in 18 patients aged 21-26 years where marked reduction in the probing depth and an improvement in the probing attachments in those cases treated with PRP, as well as the formation of new bone tissue in the bony defect.

Even though some studies suggests that the use of PRP in the alveolar socket after Tooth extractions is certainly capable of improve soft tissue healing but there is Insufficient evidence which supports the efficacy of PRP in improving bone regeneration(13,)surgical extraction of a mesioangular deeply impacted third molars. The study was conducted in 18 patients aged 21-26 years, where marked reduction in the probing depth and an improvement in the probing attachments in those cases treated with PRP, as well as the formation of new bone tissue in the bony defect even though some studies suggests that the use of PRP in the alveolar socket after tooth extractions is certainly capable of improve soft tissue healing but there is insufficient evidence which supports the efficacy of PRP in improving bone regeneration(13,).

\section{PRP IN PERIODONTAL SURGERY}

PRP plays an important role in the management of furcation defects and reconstruction Of the soft tissues (16). studies showed that platelet derived growth factor class 3 furcation defects (16).

Root coverage- incorporating the PRP/PPP technique into connective tissue grafting enhance the healing and soft tissue e regeneration .(17).

\section{Sinus lifts augmentation.}

\section{PRP IN IMPLANTOLOGY.}

PRP also used sinus graft procedure in order to obtain a dense vital in shorter interval Of time when mixed with-bovine thrombin ( autologous platelet gel). They can also play an Important role in horizontal and vertical bone augmentation. Bone to implant contact is Increased by coating the implants with PRP.(18).

4. The use of PRP has also been proposed in the management of bisphosphonate-related osteonecrosis of the jaw (BRONJ) 18)

\section{References.}

[1]. Harrison JS(1994):haemostasis .In Harrisons principles of internal medicine.

[2]. Tara - aghaloo .investigation of platelet rich plasma in rabbit cranial defects - a pilot study .journal oral maxillofacial surgery ; $2002,1176-1181$

[3]. Wirthlin RM (1989): growth substances : potential use in periodontitis .J west soc of periodontal / periodontal abstract 37:3

[4]. Dean H Whitman : an autologous alternative to fibrin glue with applications in oral and maxillofacial surgery .journal oral and maxillofacial surgery ; $1997,1294-1299$

[5]. robert E .marx et al platelet rich plasma : growth factor enhanced for bone graft .journal of oral surgery ,oral medicine ,pathology.oral radiology and endodontics 1998,638-46

[6]. Lynch et al .polypeptide growth factors : molecular mediators of tissue repair .molecular pathogenesis of periodontal disease $1994 ; 415-425$.

[7]. anitua et al ..plasma rich growth factors : preliminary results of use in preparation of future sites of implants .international journal of oral maxillofacial implants .1999;529-535.

[8]. Sanchez et el .Is platelet rich plasma a perfect enhancement factor ?a current review .international journal of oral and maxillofacial implants .2003;93-103 
[9]. Rani somani et al , platelet rich plasma- A healing aid and perfect enhancement factor : review and case report

[10]. Man D, Plosker H, Winland-Brown JE. The use of autologous platelet-rich plasma (platelet gel) and autologous platelet-poor plasma (fibrin glue) in cosmetic surgery. PlastReconstrSurg 2001; 107(1):229-37.

[11]. Weibrich et al .comparison of platelet concentration collection system with platelet rich in growth factors kit to produce platelet rich plasma a technical report .international journal of oral and maxillofacial implants .2005;118-123

[12]. Robert E Marx platelet - rich plasma .evidence to support its use .journal of oral and maxillofacial surgery $2004,489-496$

[13]. Dr. Kiran N K1, Dr. Mukunda K S2, Dr.Tilak Raj T N3 .platelet concentrates .A promising innovation in dentistry. Journal of dental science and research 2:1 pg no 50-61.

[14]. Platelet rich plasma - utilizing autologous growth factors for dental surgery to enhance bone and soft tissue grafts. New York State Dental Journal 3-02.

[15]. gillbertosammarino - use of autologous platelet rich plasma in periodontal treatment defect after extraction of impacted third molar. journal of oral and maxillofacial surgery $2005,766-770$

[16]. Park JB, Matsuura M, Han KY, Norderyd O, Lin WL, Genco RJ, and other. Periodontal regeneration in class III furcation defects of beagle dogs using guided tissue regenerative therapy with platelet-derived growth factor. J Periodontol 1995; 66(6):462-77.

[17]. Carlson NE, Roach RB: Platelet-rich plasma: clinical applications in dentistry. J Am Dent Assoc 2002, 133:1383-1386.

[18]. Nikolidakis D, Jansen JA: The biology of platelet-rich plasma and its application in oral surgery: literature review. Tissue Engineering: Part B 2008, 14:249-258. 